\title{
Emoticon Analysis with Dynamic Text based Opinion Mining
}

\author{
Sanam Kadge \\ Assistant Professor, \\ Department of \\ Information \\ Technology, M.H. \\ Saboo Siddik College \\ Of Engineering, \\ Mumbai, India
}

\author{
Saba Panchbhai \\ Student (B.E), \\ Department of \\ Information \\ Technology, M.H. \\ Saboo Siddik College \\ Of Engineering, \\ Mumbai, India
}

\author{
Sakina \\ Padghawala \\ Student (B.E), \\ Department of \\ Information \\ Technology, M.H. \\ Saboo Siddik College \\ Of Engineering, \\ Mumbai, India
}

\author{
Kausar Chhipa \\ Student (B.E), \\ Department of \\ Information \\ Technology, M.H. \\ Saboo Siddik College \\ Of Engineering, \\ Mumbai, India
}

\begin{abstract}
Social networking is a very popular communication tool where opinions are shared by millions of users thereby generating tremendous data every day. Adjectives in this data represent emotions such as good, amazing etc. for positive nature and poor, bad, inferior for negative nature. Also emoticons such as $: ;$ for happy feeling and $: ;$ for sad feeling etc are used which are necessary to be treated along with adjectives to know blogger's expression. Opinion mining is generally performed by matching words to a dictionary or a corpus that contains static set of words. However if the user uses new words which are not present in the existing dictionary, those words may be neglected. To overcome this shortcoming, this paper proposes a novel algorithm concerned with auto addition of new words at runtime thus making the dictionary dynamic. The algorithm not only gives ratings for adjectives but also for emoticons. The polarity of these newly added words will be defined by considering the polarity of its neighboring words. The analysis shows that novel algorithm gives more accurate ratings than the previous findings. These accurate ratings can help users to choose the products wisely before buying thereby benefiting the vendor as well as users.
\end{abstract}

\section{Keywords}

Opinion mining, sentiment analysis, emoticons analysis, social network mining, dynamic opinion mining

\section{INTRODUCTION}

With the rapid growth of World Wide Web and increasing ecommerce websites, people are attracted to buy products or services online. People are mostly dependent on the internet for any information or reviews about a particular product or service. As customer reviews or feedback on the web has an impact on the customer's decisions, these feedbacks have become an important source of information for businesses to take into account when developing marketing and product development plans. Due to the large number of customer reviews provided on the internet, it is difficult for potential customer to get efficient review details for a particular product and is also a challenging task. Therefore, many users are dependent on websites that provides ratings as well as a brief review about others' opinion.

Human life is filled with emotions and opinions. The world cannot be imagined without them. Emotions and opinions play a vital role in nearly all human actions. They lead the human life by influencing the way we think, what we do and how we act. Even more importantly, it has changed the way we share information [1]. To express these emotions over a particular product or service, humans tends to use the emoticons along with the opinions provided.

Today people can not only view the existing information or ratings of a particular product or service but they also share their ideas, news and knowledge with the community at large. But these existing algorithms do not consider the new words which do not exist in their dictionary. Sometimes these words might contain some very important aspects that can affect the true rating of the product. Thus this paper proposes an algorithm which will generate the rating of the products based on these new words which were not considered by earlier systems. The customer reviews provided can be in the form of text or emotions expressed with the help of emoticons. It is important to analyze these emoticons used in the users comments as that can be an important factor to provide overall rating for a particular product or service. Based on the customer reviews provided, the text and the emoticons used in these comments are evaluated by the system and the algorithm will generate an accurate rating dynamically by handling the words at runtime and classifying them as positive or negative or neutral by parsing the words in each comments. With the help of this, true rating of all the products, the popularity and usefulness of a product can be determined and can attract the targeted customers for those respective products.

\section{OPINION MINING}

Opinion mining or sentiment analysis is the use of natural language processing, text analysis and computational linguistics to identify and extract subjective information in different source materials. It is the field of study that analyses peoples' opinions, sentiments, evaluations, appraisals, attitudes, and emotions towards entities such as products, services, organizations, individuals, issues, events, topics, and their attributes [2]. Opinion Mining is a process of automatic extraction of knowledge from the data and opinion of others about some particular topic or problem. The idea of Opinion mining or Sentiment Analysis tool is to "process a set of search results for a given item, generating a list of product attributes (quality, features etc.) and aggregating opinion"[1].

In face-to-face communication, sentiment can often be deduced from visual cues like smiling. However, in plaintext computer-mediated communication, such visual cues are 
lost opinions. The emoticons are defined as visual cues used in texts to replace normal visual cues like smiling to express, stress, or disambiguate one's sentiment. Emoticons are typically made up of typographical symbols such as ":.", "=", "-", ")", or "(" and commonly represent facial expressions. Emoticons can be read either sideways, like ":-(" (a sad face), or normally, like "( $\left.{ }^{\wedge}\right)$ " (a happy face). In recent years, several approaches to sentiment analysis of natural language text have been proposed. Many state- of-the-art approaches represent text as a bag of words, i.e., an unordered collection of the words occurring in a text. Such an approach allows for vector representations of text, enabling the use of machine learning techniques for classifying the polarity of text. Features in such representations may be, e.g., words or parts of words [3]

\subsection{Need for opinion mining}

Textual information in the world can be broadly classified into two main categories, facts and opinions. Facts are objective statements about entities and events in the world. Opinions are subjective statements that reflect people's sentiments or perceptions about the entities and events. Much of the existing research on text information processing has been (almost exclusively) focused on mining and retrieval of factual information, e.g., information retrieval, Web search, and many other text mining and natural language processing tasks. Little work has been done on the processing of opinions until only recently. Yet, opinions are so important that whenever one needs to make a decision one wants to hear others' opinions [3]. Thus the need of opinion mining arises.

Decision making both on individual and organizational level is always accompanied by the search of other's opinion on the same. With tremendous establishment of opinion rich resources like, reviews, forum discussions, blogs, microblogs, Twitter etc provide a rich anthology of sentiments. This user generated content can serve as a benefaction to market if the semantic orientations are deliberated [4]. Sentiment analysis, which is also called opinion mining, involves in building a system to collect and examine opinions about the product made in blog posts, comments, reviews or tweets. Sentiment analysis can be useful in several ways. For example, in marketing it helps in judging the success of an ad campaign or new product launch, determine which versions of a product or service are popular and even identify which demographics like or dislike particular features [5]. Thus opinion mining can greatly help for marketing purpose. The evolution of automated systems and digital information in every field of life is evolving rapidly which tends to generate data. As a result huge volumes of data are produced in field of science, engineering, medical, marketing, finance, demographic etc. Automated systems are meant to automate analysis, summarization and classification of data and number of efficient ways is available to store huge volumes of data [6]. Opinion mining thereby provides the base for such systems.

\subsection{Text Mining and Emoticon Analysis}

Text mining deals with the mining of the text in the users based comments. Opinion Mining is extracting the opinions from text. Opinion Mining can be applicable to any kind of text. Gathering feeling or emotions associated with text is known as Opinion Mining. Mining of the text is important and useful for providing ratings, to know the users experience for a particular or service, for any event or place, for a movie, hotel, book, etc. Mining is also useful to provide opinion on a particular product or service. Written Text is one good source for expressing your ideas, emotions and feelings. Human Being has a power to feel different kinds of emotion because life of every human being is filled with a lot of emotions. Joy, fear, anger and sadness are few emotional states that a person encounters in day to day life. And using computer, the categorization of text in these emotional states is known as sentimental analysis/emotion detection. There is a minor difference between Sentiment Analysis and emotion detection. In general, Sentiment Analysis divides text into two binary states (positive/ negative) whereas Emotion Detection uses larger set of emotional states like joy, fear, anger, brief, surprise or disgust for division of text [7]. The customer reviews is provided in the form of text or emotions expressed with the help of emoticons. Various emotional states like joy, fear, anger, brief, surprise or disgust, sadness etc. are expressed with the help of different emoticons. The analysis of these emoticons is important in opinion mining as it can be an important factor to provide overall rating for a particular product or service. Sometimes user review is provided only with the help of the emoticons so emoticon analysis becomes important. The user based comments in the form of text are classified into positive or negative or neutral dictionaries and comments in the form of emoticons are classified into positive or negative by parsing the words in each comment. Each parsed word is then matched with the dictionary, if the match is found in any of the positive or negative or neutral dictionaries then the count increases else if the match is not found then it is auto-added in the neutral dictionary and the polarity of these auto added words can be determined with the help of considering the polarity of its neighboring words.

\section{EXISTING RESEARCH WORK}

As mentioned above existing systems are based either purely on opinion mining/web mining/text mining/sentiment analysis or they can also be based on text mining combined with emoticon analysis. In the existing systems the text mining is done on the basis of the words that are present in the dictionary, thus making the dictionary static. Polarity is assigned to only to these words thus classifying them as positive, negative or neutral. Text mining is done based on the algorithms used like naïve bayes, tscan, svm, jacccard clustering and many more.

\subsection{Modified TSCAN algorithm}

The latest algorithm that had been used for text mining was a modified version of tscan also called modified tscan. The tscan algorithm technique overcomes the inefficiency of reading the full content in the online resources and it performs the sentence reading from left to right.

\subsection{Finite State Machine}

The emoticon analysis technique uses a Finite State Machine (FSM) to detect the emoticons polarity.

A Finite State Machine (FSM) is a basically a state machine used to design computer programs based on certain logic. The machine has a start state, which takes one input at a time and moves to another state. It remains in one state at a time. There can be many final states. Final state indicates an output [8]. Figure 1 depicts the Finite State Machine to determine the emoticons polarity. 


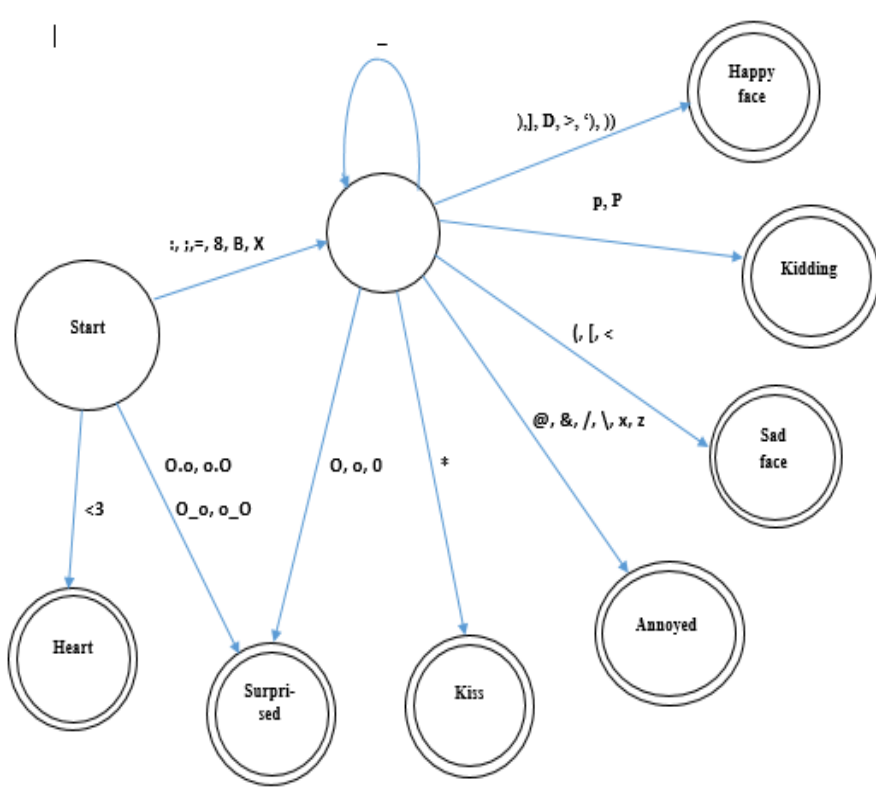

Figure 1. Finite State Machine

\section{PROBLEMS IN THE EXISTING SYSTEM}

Social web analysis is all about the users because of whom a large amount of content is generated every day. This content is rapidly changing with updates on a daily basis [9]. The analysis of this content is important since it provides us the opinions based on the user reviews. The opinions are provided in the textual form along with the emoticons. Textual sentimental analysis classifies the reviews as positive or negative or neutral. Although, the user reviews are classified as either positive or negative, there is a problem in finding the opinion holder's likes or dislikes. A positive opinion on an object does not mean that the user has positive opinions on all aspects or features of the product. Likewise, a negative opinion does not mean that the user dislikes everything about the product.

Also all the existing systems work on a dictionary which is static. The novel algorithm will build a dynamic dictionary. Static dictionary implies that the opinion is extracted only from the words which are pre mentioned in the existing algorithms. This can be a problem since a new word may hold great importance in defining the opinion of a person and thereby providing the wrong rating.

A word can be considered as positive in one situation and negative in another situation. This can be difficult as a sentence can be considered negative because of the use of negative words in it. Sarcastic sentences exist in text. In such a scenario, positive words can be used in a negative sense. So, text in a statement can be hard to identify because of the sarcasm used in it. People are more likely to combine different opinions in the same sentences. Such sentences can be difficult to parse for opinion mining.

Another challenge faced by opinion mining is the domain dependent nature of words holding sentiments. Some features may give very good output in one domain, at the same time poor output in some other domain [11]. There can be another problem which relates to how the words are spelt by different people. For example, many people write the word "awesome" as "awsum", "good" as "gud" etc. This acts as a challenge to correctly process the language and find out the polarity of the sentence. Also, emoticons give a insight of the sentence or text [8]. Thus emoticons are also important to consider. In the existing system the sentimental analysis is done only on the basis of the words present in the dictionary and based on these words polarity as assigned to them. There was no autoadding facility for the new words. So the new words entered by the user were not considered which might be important in rating of the overall comment. Also most of the existing systems were only based on text mining without consideration of emoticons.

Another problem in the existing system is that nowadays people use all types of languages while commenting on the sites. These languages are again not included in a static dictionary. This paper overcomes this problem by using the novel algorithm.

\section{PROPOSED SYSTEM}

By addressing some of the problems in the existing system, a dynamic approach has been proposed. In this dynamic opinion mining approach, a novel algorithm is proposed for auto-adding feature. The auto-adding facility helps to add the new words to the existing neutral dictionary, thus making the dictionary dynamic. Hence the new words entered by the users are considered along with the existing words for the overall rating of the product. The proposed system module is shown in figure 2 .

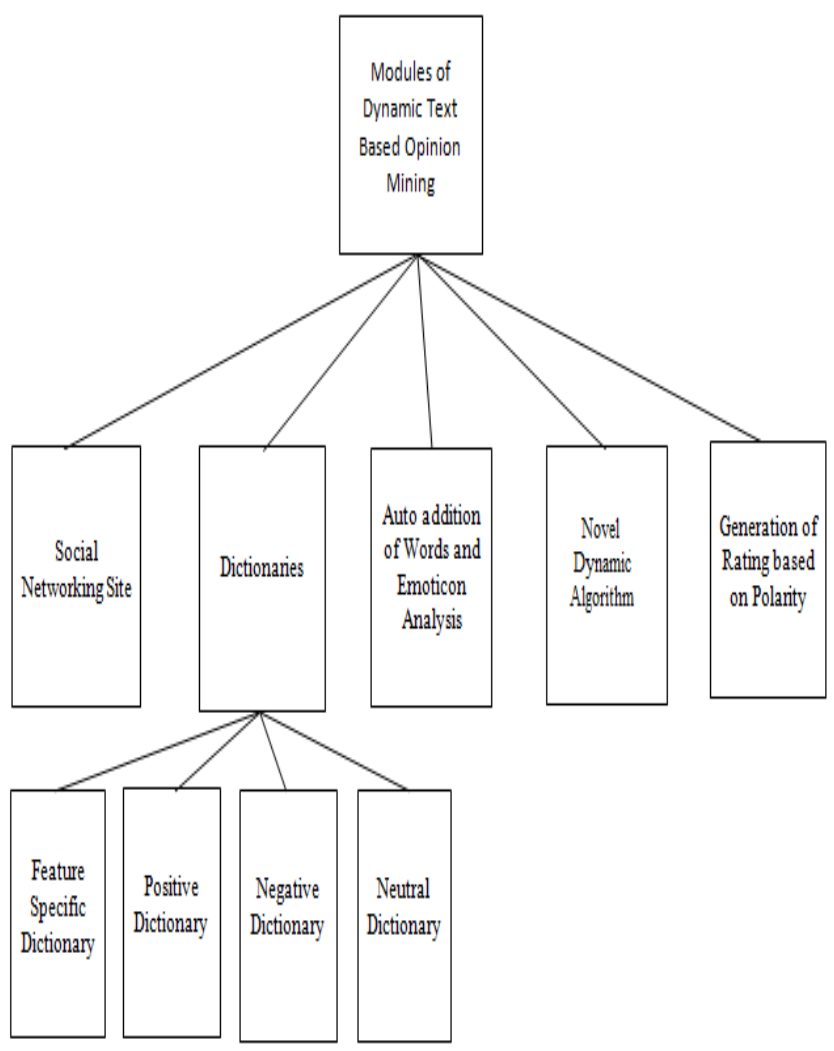

Figure 2. Proposed system module 


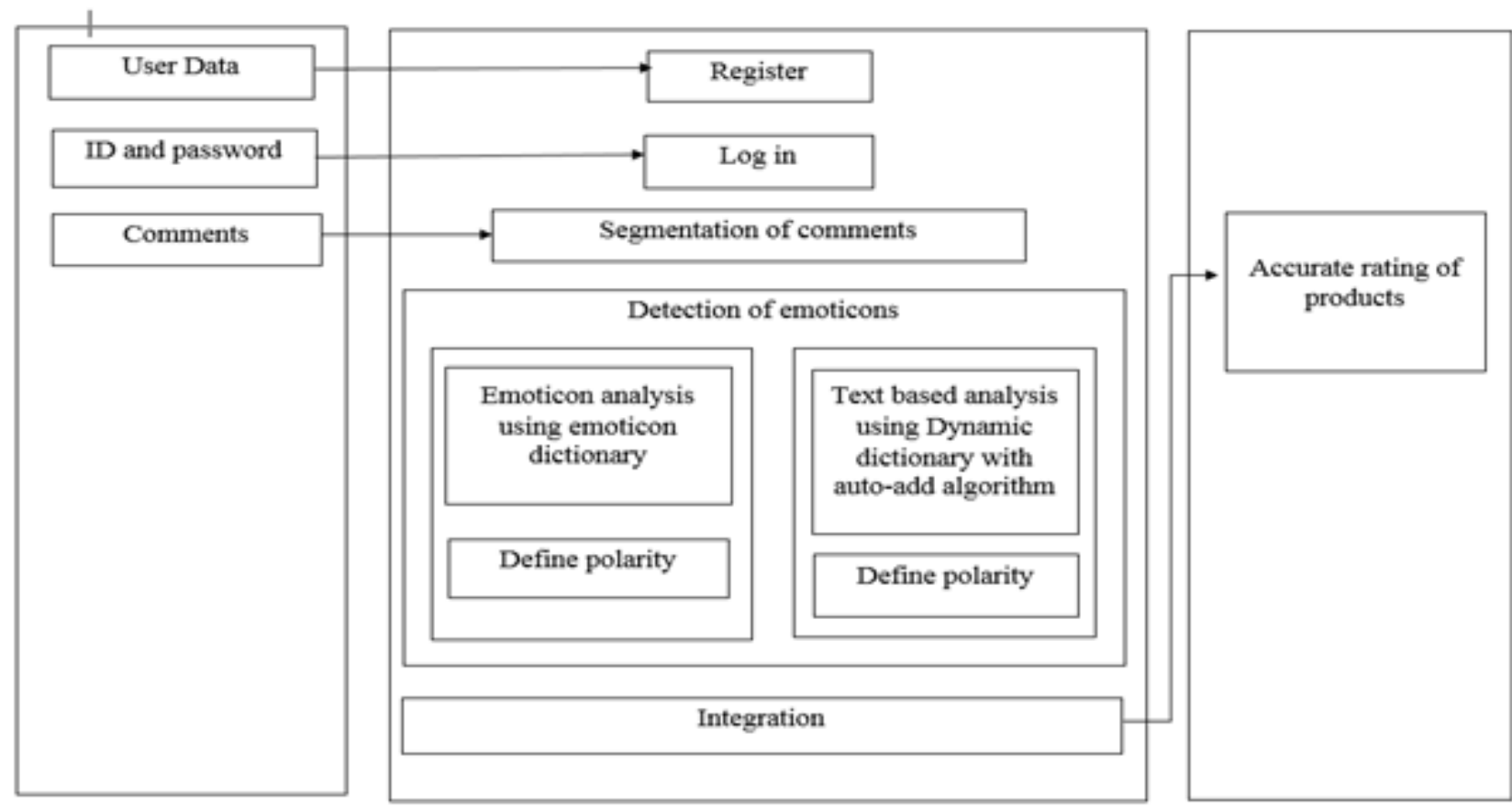

Input

Processing

Output

Figure 3. Block Diagram

The proposed system consists of different modules as shown in the diagram Figure 2. There are four types of dictionaries like feature specific dictionary, positive dictionary, negative dictionary and neutral dictionary. There is also a module based on auto addition of words and emoticon analysis, dynamic opinion mining algorithm and on rating.

The purpose of this system is to auto add the new words during runtime and classify them thus making the dictionary dynamic. It will also classify the emoticons as positive or negative that are used to express emotions of human beings. Emoticons are classified as happy, sad, angry, afraid, fear, disgust, sleepy, surprised, etc. in the pre-existing dictionary and based on this polarity is assigned. The auto-adding feature allows the system to add new words to the neutral dictionary thereby making the dictionary dynamic and polarity will be assigned to the new words based on the neighboring words and also it will be able to classify the new words into positive, negative or neutral based on the user comments. The polarity count for each word or new word will be stored in the database and based on this count the more accurate ratings will be generated by calculating the difference between the positive and negative counts. If there is any new word to be added then an auto add algorithm will make sure that it is added to the database and classified for the purpose of rating. This Information System provides the facility of Dynamic Opinion Mining. The dynamic dictionary is provided only for auto-adding the new words to the dictionary and not for the emoticons. The accurate rating for the products is generated based on the reviews of the user comments. The above diagram Figure 3 shows the block diagram. In the above Figure 3, it depicts the input, processing and output stages for the system. The input stage considers the user data, login process and comments entered by the user. The processing stage shows how the system generates the rating. The output stage displays the accurate rating generated by the dynamic algorithm.

The workflow of the system is as shown in the diagram Figure 4. The web users should register or login in the system to provide comments for a particular product or service. The user comments is then segmented, divided into text segments and then preprocessing is performed on each of the comments. Each word in the comment is parsed during preprocessing process. The polarity is defined for both emoticon as well as text based analysis. In text based analysis, auto-adding of new words feature is provided so that ne words are added during the runtime and its polarity can be determined based on its neighboring words. Three types of dictionaries are specified that is Positive, Negative and Neutral, each of them describing respective polarity. The polarity count for each word is maintained in the database based on its occurrence and overall positive or negative count is taken into consideration for determining the overall rating for the product. Thus it helps in generating a more accurate rating for the respective product. 


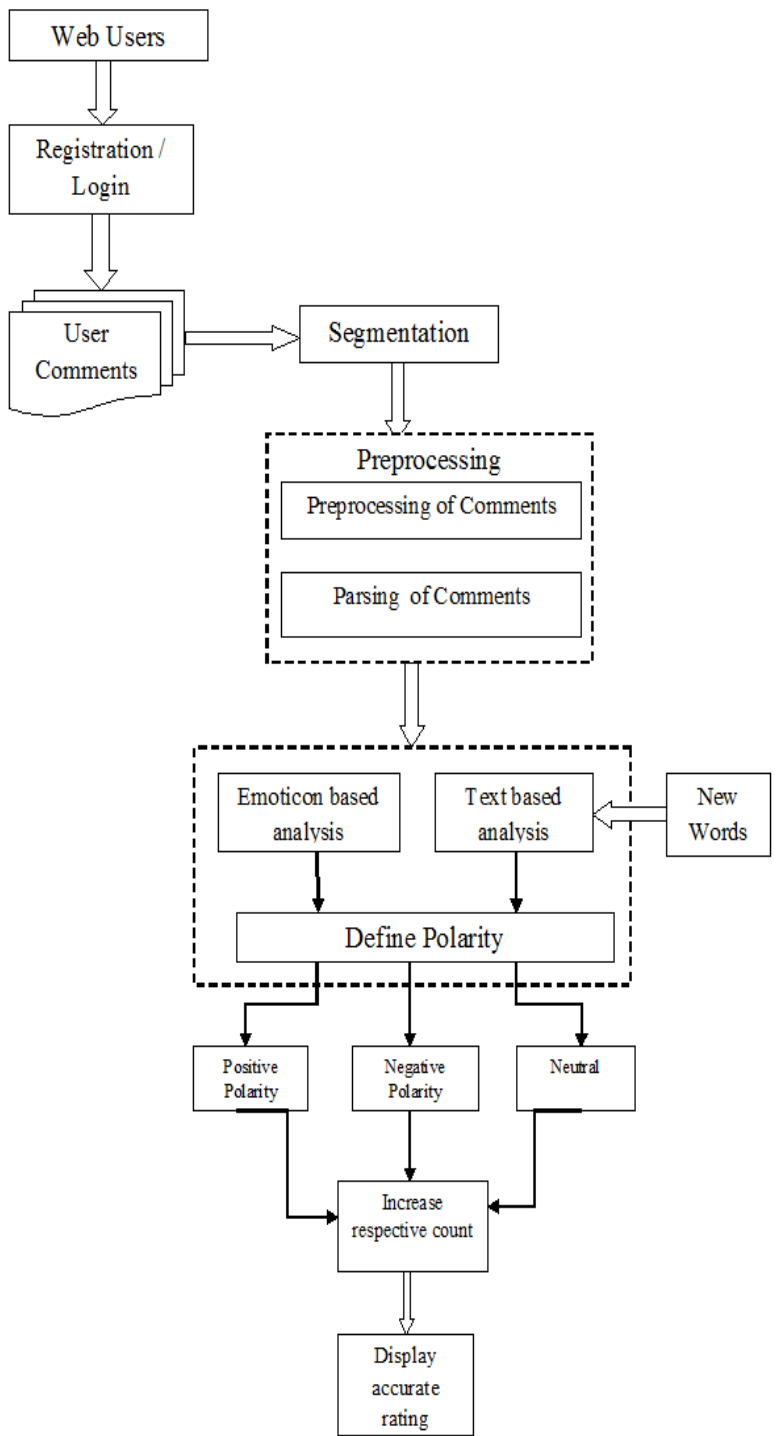

Figure 4. Workflow of the System.

The proposed system will have two types of users, registered user and unregistered user. Unregistered user can only read the other users comments and can view the products and product specification. The registered user can view the products as well as give comments and rating for a particular product. It is necessary to login the system in order to comment for any particular product. The privilege of being a registered user is that he gets suggestions for products based on his search history and the product he/she searches are visible in recently viewed section.

Each word in the comment given by user is parsed and polarity for the comment is determined. The polarity count for each word is maintained in the database based on its occurrence and overall positive or negative count is taken into consideration for determining the overall rating for the product.

The polarity count for emoticons and text based analysis is taken into consideration for determining the overall rating of a product.

\section{IMPLEMENTATION}

For implementation purpose, this paper proposes a novel algorithm for dynamic opinion mining. The algorithm describes the preprocessing of comments, defining the polarity of emoticons with the help of dictionaries, autoadding of new words in the neutral dictionary, defining its polarity based on neighboring words and rating generation. The algorithm is applied on all the user comments to identify the polarity of words for rating purpose. The algorithm analyzes the comments word by word, identifies the polarity of these words and stores the count in the database. The rating is provided in numerical form in the scale of 1 to 10 . For rating purpose, the overall count is taken into consideration, that is overall count of both positive and negative polarity. If the overall count for positive polarity is more than the negative polarity, then positive rating is provided else if the negative polarity count is more than the positive polarity count then negative rating is provided. If both the counts are equal then moderate rating is provided.

\subsection{Sample Dictionaries}

For rating purpose, it is necessary to define the polarity of each user comments. The polarity defined for the comments can be positive or negative or neutral based on the set of words described in the dictionaries. Here four set of dictionaries named as Positive dictionary, Negative dictionary, Neutral dictionary and Feature specific dictionary are defined. The given table below shows the sample words existing in these dictionaries.

Table 1: Set of Dictionaries Specified

\begin{tabular}{|c|l|}
\hline \multirow{2}{*}{ Positive } & $\begin{array}{l}\text { Brightens, Great, Awesome, Good, Cool, } \\
\text { Fun, Nice, Superb, Best, Excellent, Fast, } \\
\text { Beautiful, Grand, Powerful, Solid, Sleek, Thin, } \\
\text { Light, Popular, Lovely, Brilliant, Long, } \\
\text { Beauty, Wonderful, Super, Incredible, Fantastic, } \\
\text { Flawless, Happy, Smooth. }\end{array}$ \\
\hline $\begin{array}{c}\text { Negative } \\
\text { Words }\end{array}$ & $\begin{array}{l}\text { Lags, Lacks, Difficult, Doesn't, Faulty, Sucks, } \\
\text { Cheap, Cannot, Hesitating, Bad } \\
\text { Kinks, Slow, Slippery, Disappointed, Never } \\
\text { Cheaters, Blame, Poor, Inappropriate } \\
\text { Disagree, Sad, Avoid, Worst, Below } \\
\text { Pathetic, Drains. }\end{array}$ \\
\hline $\begin{array}{c}\text { Neutral } \\
\text { Words }\end{array}$ & $\begin{array}{l}\text { Very, Worth, Issue, Score, Trust, Default, } \\
\text { Complaints, Buy, wife, man. }\end{array}$ \\
\hline $\begin{array}{c}\text { Feature } \\
\text { Specific } \\
\text { Words }\end{array}$ & $\begin{array}{l}\text { Phone, Front Camera, Back Camera, Display, } \\
\text { Memory, Volume, Battery Life, RAM, }\end{array}$ \\
& Processor, Screen, Quality, Image. \\
\hline
\end{tabular}

\subsection{Algorithm}

The following steps of the algorithm is as shown below:

Consider 1 comment at a time.

\section{Step 1: Preprocessing}

Remove punctuations like + \# \&@ etc.

Remove words that are not required for rating purpose and has no effect on the opinion of a person. These words include and, is, her, this, that, for, of, etc. 
Also remove words which are specifying features of a particular product so that the unnecessary processing of those words is not done. For example if your product is a mobile phone then the feature specific words will include camera, clarity, display etc.

\section{Step 2: Emoticon Analysis}

Parse the sentence and check for emoticons. If an emoticon exists then the polarity count is considered as per the emoticon.

Table 2: Examples of Positive and Negative Emoticon Icons.

\begin{tabular}{|c|c|}
\hline Positive emoticons & 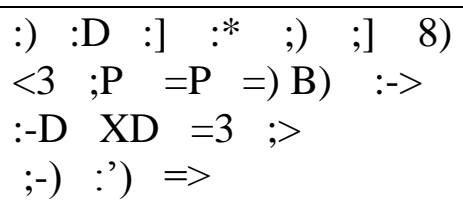 \\
\hline Negative emoticons & 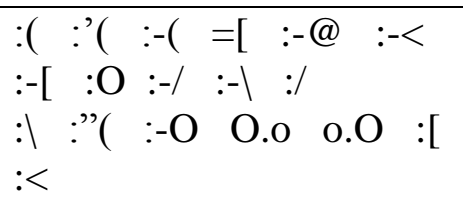 \\
\hline
\end{tabular}

\section{Step 3: Dynamic Algorithm}

The algorithm will parse one word at a time

The algorithm considers 3 dictionaries after preprocessing namely- positive words, negative words and neutral words.

If the word is a match in of the 3 dictionaries then the polarity to that word will be assigned accordingly. If word is positive then the positive variable word count increases and for negative word the negative variable word count increases. If the word is not a match it will be auto added in the neutral dictionary by an auto adding algorithm. For the newly added word check the polarity of the neighboring words of that comment and accordingly consider the polarity count of that word. That is if neighboring words are negative then the negative count is considered else positive.

For each occurrence of the newly added word again increase the polarity count variable based on neighboring words of that comment. Maintain the polarity count for each occurrence of that word that is how many times that word was considered as either positive or negative.

When any of the positive or negative count for that word crosses 5 then it should be added to the respective dictionary and deleted from the neutral dictionary.

\section{Step 4: Rating Generation}

The above data along with emoticon count will be saved in specific variables.

Repeat steps 1, 2 and 3 for all the comments.

The positive count of all the words of all the comments is then stored in a final positive count variable and the negative count of all the words of all the comments is then stored in a final negative count variable.

The difference between these variables is calculated and a rating meter provides the rating on the scale of 1 to 10 .

\subsection{Screens of "view your review"application}

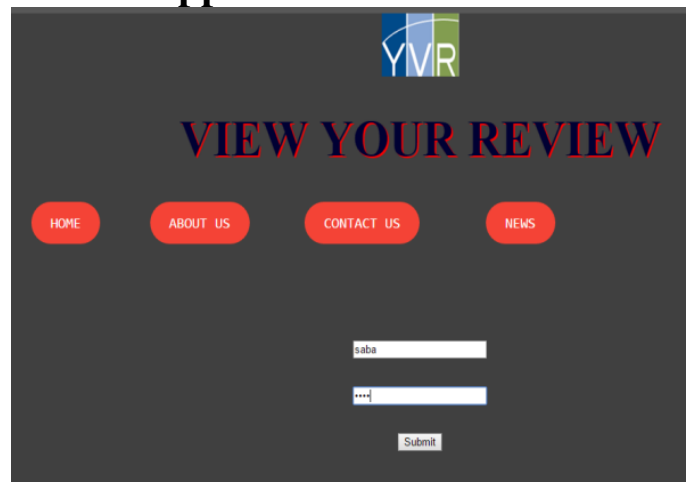

Figure 5. Login screen for the user

In the above figure 5 the user first needs to register or sign up. After the user has been registered then the user can login to get access to the commenting system. It should be noted that the user can only comment if he or she has logged in. without logging in the user can see the reviews and ratings but not comment. Thus the login becomes an important part of the system.

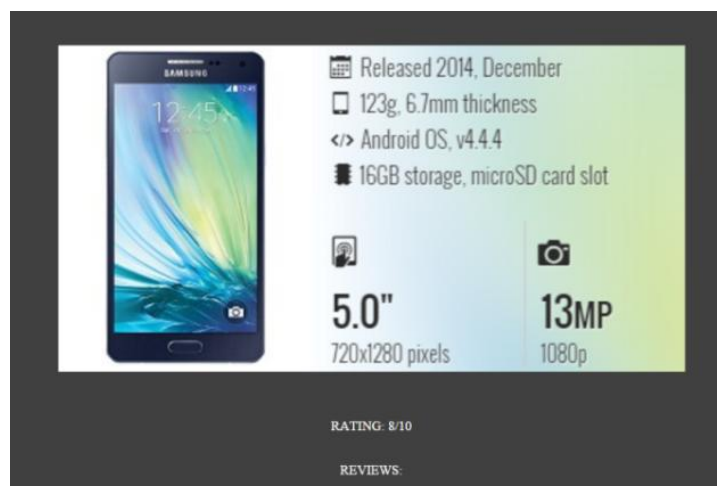

Figure 6. Product and its rating displayed

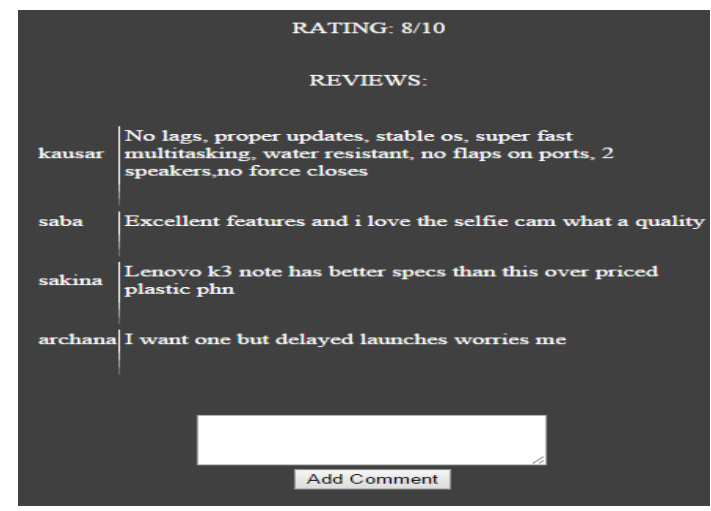

Figure 7. Ratings, reviews and commenting facility

In the above figure 6 and 7 it is clear that ratings are provided as per the reviews of the users based on the mobile phone. At any point of time the user can add a new comment which will be stored in the system and the rating will be affected accordingly. This rating system will use the novel algorithm as mentioned before. 


\section{ANALYSIS OF RESULTS}

\subsection{Comment analysis}

In this paper, analysis is done on some of the users comments from Amazon.com.

Considering the following comments for a particular mobile device:

Table 3: Example of comments from amazon.com

\begin{tabular}{|c|c|c|}
\hline $\begin{array}{c}\text { Example } \\
\text { No. }\end{array}$ & COMMENTS & $\begin{array}{c}\text { POLARITY } \\
\text { COUNT }\end{array}$ \\
\hline 1. & Very good phone with front \\
camera and amoled display & +1 \\
\hline 2. & Very slow phone & -1 \\
\hline 3. & Good vis-à-vis price & +2 \\
\hline 4. & $\begin{array}{c}\text { Really good and brightens up my } \\
\text { kindle with cute display :D }\end{array}$ & +4 \\
\hline 5. & Not worth the price. & -1 \\
\hline & Net polarity count: & 5 \\
\hline
\end{tabular}

In the above comments;

In the first comment 'good' is positive. 'With' and 'and' are eliminated during preprocessing. 'Phone', 'front camera', 'amoled', and 'display' belong to the feature specific dictionary. Thus it makes the net polarity of that comment as +1 .

In the second comment 'slow' is negative, 'phone' is feature specific and 'very' belongs to the neutral dictionary. Thus it makes the net polarity of that comment as -1 .

In the third comment 'good' is positive, 'price' is feature specific. Here 'vis-a-vis' is a new word which will be first auto added to the neutral dictionary. Followed by this the polarity of this newly added word is determined based on the neighboring words. Here the neighboring word 'good' is positive so the polarity of 'vis-a-vis' is considered positive. Thus it makes the net polarity of that comment as +2 .

In the fourth comment 'good', 'cute', 'brightens' are positive. 'With', 'up', 'my' and 'and' are eliminated during preprocessing. 'kindle' and 'display' belong to the feature specific dictionary. Since we have a smiley here that is positive, the emoticon analysis leads to a +1 count. Thus it makes the net polarity of that comment as +4

In the fifth comment 'not' is negative, 'price' is feature specific and 'worth' belongs to the neutral dictionary. Thus it makes the net polarity of that comment as -1 .

Analyzing these five comments, the net polarity count of the comments sums up to +5 .

The result of the analysis is as shown above in the table. The resultant analysis indicates that different people uses different words, sarcastic words along with the use of emoticons to express their emotion over a particular product. Emotions are usually expressed in the form of emoticons when a user is either too happy or too sad after using a particular product. The different sarcastic words used in the comments given by the users is sometimes difficult to analyze, hence to find more accurate rating of a product becomes a difficult task. Although this paper is trying to overcome this, but all the sarcastic words, cannot be overcome. The net polarity count in the table is the difference between the positive polarity count and negative polarity count.

\subsection{Accuracy of results}

To find the accuracy of the novel algorithm this paper compares the rating algorithm used by flipkart and amazon to that of the novel algorithm. The sample comments were taken from flipkart as well as from amazon and novel algorithm was applied to it, to obtain the rating. The result observed in the graph below shows that where flipkart had provided a rating of 5 , our algorithm provided rating of 4.7 because of the negative points being considered by the novel algorithm. Similarly in the case of figure 9 where we obtain accuracy by comparing novel algorithm with amazon's algorithm. The novel algorithm in second case produces a rating of 4 whereas amazon gives a rating of 4.6 .

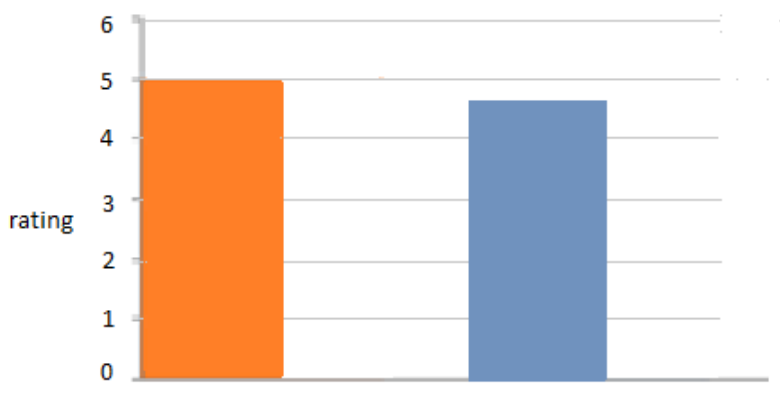

Flipkart Algorithm Novel Algorithm

Figure 8. Comparison of Novel Algorithm with Flipkart

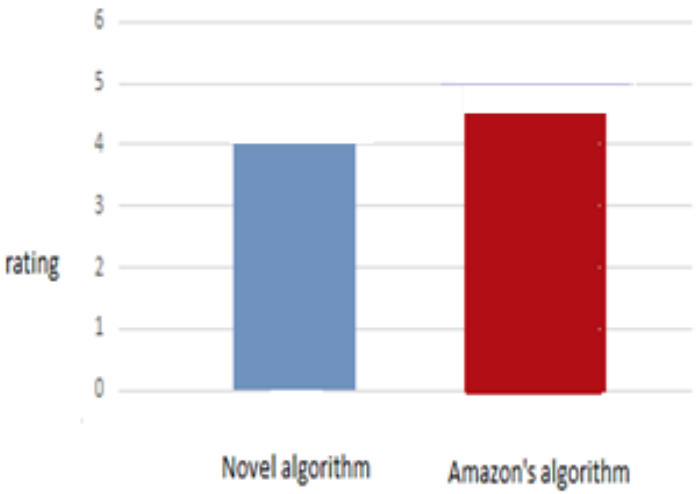

Figure s 9. Comparison of Novel Algorithm with Amazon

In the above figure $8 \&$ figure 9 the ratings provided by Flipkart and Amazon have been compared for a particular product with the novel algorithm and observed that novel algorithm provided more accurate ratings.

\section{CONCLUSION}

Opinion mining is an emerging field of data mining used to extract the knowledge from huge volumes of data that may be customer comments, feedback and reviews on any product or topic etc. As people increasingly use emoticons to express 
their opinions, it is important for the automated system to correctly interpret these emoticons for rating purpose. The key contribution of our work lies in the dynamic algorithm and analysis of emoticons that plays an important role in conveying the users comments or opinions. With the existing system based on static dictionary, the dictionary used in the proposed system is dynamic based on the dynamic algorithm that will add new words during runtime. In this paper a novel algorithm has been proposed that determines the rating of a product based on the user comments in an accurate way using a dynamic approach. This helps in overcoming the drawback thus providing more efficient accurate rating for the products.

\section{REFERENCES}

[1] S Padmaja and Prof. S Sameen Fatima,February 2013"Opinion Mining and Sentiment Analysis -An Assessment of Peoples' Belief: A Survey"International Journal of Ad hoc, Sensor \& Ubiquitous Computing (IJASUC) Vol.4, No.1,

[2] Pratik N. Kalamkar, Anupama G. Phakatkar, "Review Based Entity Ranking using Fuzzy Logic Algorithmic Approach: Analysis".

[3] Bing Liu Department of Computer Science University of Illinois at Chicago $851 \mathrm{~S}$. Morgan Street Chicago, IL 60607-0753 "OPINION MINING".

[4] Pravesh Kumar Singh1, MohdShahid Husain2, international journal on soft computing (ijsc) vol. 5, no. 1, february 2014 doi: $10.5121 /$ ijsc.2014.5102 11 methodological study of opinion mining and sentiment analysis techniques.

[5] Volume 2, Issue 6, June 2012 ISSN: 2277 128X International Journal of Advanced Research in Computer
Science and Software Engineering Research Paper Available online at: www.ijarcsse.com "Sentiment Analysis and Opinion Mining: A Survey

[6] G.Angulakshmi1, Dr.R.ManickaChezian2, International Journal of Advanced Research in Computer and Communication Engineering Vol. 3, Issue 7, July 2014 Copyright to IJARCCE www.ijarcce.com 7483 An Analysis on Opinion Mining: Techniques and Tools.

[7] Jasleen Kaur, Jatinderkumar R. Saini, Emotion Detection and Sentiment Analysis in Text Corpus: A Differential Study with Informal and Formal Writing Styles.

[8] Geetika Vashisht1, Sangharsh Thakur, International Journal of Emerging Technology and Advanced Engineering Website: www.ijetae.com (ISSN 2250-2459, ISO 9001:2008 Certified Journal, Volume 4, Issue 5, May 2014) Facebook as a Corpus for Emoticons-Based Sentiment Analysis.

[9] Diana Maynard, Kalina Bontcheva, Dominic Rout, "Challenges in developing opinion mining tools for social media".

[10] Tanvir Ahmad , Mohammad Najmud Doja, July 2012 "Ranking System for Opinion Mining of Features from Review Documents"

[11] Farouque Azam, Bakhtawar Seerat, July 2012 "Opinion Mining: Issues and Challenges (A survey)",

[12] Jayashri Khairnar, Mayura Kinikar , "Machine Learning Algorithms for Opinion Mining and Sentiment Classification".

[13] Alexander Hogenboom, Daniella Bal, Flavius Frasincar, "Exploiting Emoticons in Sentiment Analysis". 